Pacific Journal of Mathematic 


\title{
CONVERGENCE TOPOLOGIES FOR MEASURES AND THE EXISTENCE OF TRANSITION PROBABILITIES
}

\author{
SOLOMON LEADER
}

1. Introduction. A recent approach to measure theory is the introduction of measures as functionals on spaces of continuous functions [2]. To the probabilist, however, the measures are of primary concern, with the functions occurring as integrands playing a secondary role as random variables. We are thus motivated to reverse the modern procedure. We shall introduce various topologies into spaces of measures and shall in each case investigate the dual space consisting of all continuous linear functionals on the measures. From this point of view the continuous functions form only one of many possible dual spaces to a space of measures.

The study of the dual spaces yields a necessary and sufficient continuity condition for the existence of transition probabilities in a stochastic semigroup, thus solving a problem posed by W. Feller.

We introduce topologies through the convergence of nets [5], an elegant device for analysis. The spaces of measures considered are vector spaces and usually vector lattices [1]. We admit any topology for which the vector operations are continuous, but do not require that the lattice operations be continuous.

Let $\mathfrak{Y}$ be a Boolean algebra of sets in an abstract space $X$. Wherever $\mathfrak{U}$ is required to be a $\sigma$-algebra, it shall be denoted by $\mathfrak{U}_{\sigma} \cdot \mathrm{A}$ partition $\rho$ of $X$ is a finite collection $\left\{E_{k}\right\}$ of sets in $\mathfrak{U}$ which form a disjoint covering of $X$. The partitions of $X$ form a lattice [1] if we define $\rho<\rho^{\prime}$ whenever $\rho^{\prime}$ is a refinement of $\rho$. In this way the partitions $\rho$ will be used extensively as directed indices for nets.

For each $x$ in $X$ define the unit point mass $H_{x}$ by

$$
H_{x}(E)= \begin{cases}0 & \text { if } x \text { is not in } E . \\ 1 & \text { if } x \text { is in } E .\end{cases}
$$

Such $H_{x}$ will belong to all spaces of measures considered below. A discrete measure is any finite linear combination of point masses. We shall use the symbol $E$ to denote the characteristic function of a set $E$, since the context will serve to distinguish between the set and its characteristic function. Thus,

Received May 25, 1954. This work has its origin in W. Feller's O.O.R. project at Princeton University and was supported by the Research Council of Rutgers University. 


$$
E(x)=H_{x}(E) .
$$

A step function is any finite linear combination of characteristic functions of sets in $\mathfrak{A}$. For each step function $f$ there exists a partition $\rho=\left\{E_{k}\right\}$ such that

$$
f(x)=\sum_{\rho} a_{k} E_{k}(x) .
$$

If $f$ is any real-valued function on $X, f(E)$ will denote the set of all $f(x)$ for $x$ in $\mathrm{E}$. Bars will be used to denote the diameter of a set of numbers. Thus,

$$
|f(E)|=\sup f(E)-\inf f(E) .
$$

2. The topology of simple convergence. Let $S$ consist of all finitely additive functions on $\mathfrak{A}$. That is, $F$ belongs to $S$ if

$$
\begin{aligned}
& -\infty<F(E)<\infty \quad \text { for all } E \text { in } \mathfrak{Y} \text {, and } \\
& F(A+E)=F(A)+F(E) \quad \text { for } A \text { and } E \text { disjoint. }
\end{aligned}
$$

$S$ is a vector space with the obvious definitions of addition and scalar multiplication.

Let $\left\{F_{\alpha}\right\}$ be a net in $S$. We induce a topology in $S$ by defining: $F_{\alpha}$ converges simply to $F$ if $\lim _{\alpha} F_{\alpha}(E)=F(E)$ for each $E$ in $\mathfrak{H}$.

THEOREM 1. The discrete measures are dense in S. In particular,

$$
F=\int_{X} H_{x} d F(x)
$$

where the latter integral is defined to be $\lim _{\rho} \sum_{\rho} F\left(E_{k}\right) H_{x_{k}}$ with $\rho=\left\{E_{k}\right\}$ and $x_{k}$ in $E_{k}$. $S^{*}$, the dual space of $S$, consists of all step functions (1.3). With the topology of stepwise convergence in $S^{*}$, defined below, $S^{* *}=S$.

Proof. For $\rho$ fine enough to partition $E$ and its complement $E^{\prime}$, $\sum_{\rho} F\left(E_{k}\right) H_{x_{k}}(E)=F(E)$. Hence, (2.3).

Each $\phi$ in $S^{*}$ defines a function $f$ on $X$ through

$$
f(x)=\phi\left(H_{x}\right) \quad \text { for all } x \text { in } X .
$$

Suppose $f$ were not a step function. Then, for each partition $\rho$ we could choose $x_{\rho}$ and $y_{\rho}$ in some set $E_{k}$ in $\rho$ such that $f\left(x_{\rho}\right) \neq f\left(y_{\rho}\right)$. Define 


$$
G_{\rho}=\frac{1}{f\left(x_{\rho}\right)-f\left(y_{\rho}\right)}\left(H_{x_{\rho}}-H_{y_{\rho}}\right) .
$$

Then, $\phi\left(G_{\rho}\right)=1$ for all $\rho$. But, for all $\rho \geq\left\{E, E^{\prime}\right\}, G_{\rho}(E)=0$. Hence, $G_{\rho}$ converges simply to 0 . Since $\phi$ is continuous, $\lim \phi\left(G_{\rho}\right)=0$, a contradiction. So $f$ must be of the form (1.3). Then, of course,

$$
\phi(F)=\sum_{\rho} a_{k} F\left(E_{k}\right)=\int f d F .
$$

A sequence of step functions $f_{n}$ converges step-wise to a step function $f$, if there exists a partition $\rho$ such that eventually

$$
f_{n}(x)=\sum_{\rho} a_{n k} E_{k}(x)
$$

and

$$
f(x)=\sum_{\rho} \lim _{n} a_{n k} E_{k}(x) .
$$

In this topology, every linear functional on $S^{*}$ is continuous. Since every linear functional on $S^{*}$ defines an additive function on $\mathfrak{A}$ with a unique linear extension to $S^{*}, S=S^{* *}$.

3. The topology of bounded convergence. The functions in $S$ that are bounded form the space $B$. The condition (2.1) is strengthened to

$$
-M<F(E)<M \quad \text { for all } E \text {. }
$$

The usual topology for $B$ is the topology of uniform convergence, that is, the topology induced by the norm

$$
\|F\|=\sup _{E} F(E)-F\left(E^{\prime}\right)=\lim _{\rho} \sum_{\rho}\left|F\left(E_{k}\right)\right| .
$$

With this norm $B$ is a Banach space [1]. The norm topology is defective in that there may exist bounded (that is, continuous) linear functionals on $B$ which have no representation as integrable functions on $X$, and $B$ is not reflexive.

These defects are removed by introducing the topology of bounded convergence: $F_{a}$ converges boundedly to $F$ if $\lim F_{a}(E)=F(E)$ for each $E$ in $\mathfrak{H}$, and $\left|F_{\alpha}(E)\right|<M$ for all $\alpha \geqq \alpha_{0}$ and all $E$ in $\mathfrak{A}$.

THEOREM 2. In the topology of bounded convergence the discrete measures are dense in $B$. In particular,

$$
F=\int_{X} H_{x} d F(x)
$$

defined as in (2.3). The dual space $B^{*}$ consists of uniform limits of 
step functions (1.3). That is, to every continuous linear functional $\phi$ there corresponds biuniquely a function $f$ on $X$ such that $f$ is a uniform limit of step functions and, for all $F$ in $B$,

$$
\phi(F)=\int_{X} f(x) d F(x) .
$$

With the topology of uniform convergence on $B^{*}, B^{* *}=B$.

Proof. By Theorem 1, (3.3) holds for simple convergence. Since $\left|\sum_{p} F\left(E_{k}\right) H_{x_{k}}(E)\right| \leqq \sum_{p}\left|F\left(E_{k}\right)\right| \leqq\|F\|$, the convergence is bounded for $F$ in $B$.

For $\phi$ in $B^{*}$ let $f$ be defined by (2.4). Now, suppose $f$ were not a uniform limit of step functions. Then there would exist some $\varepsilon>0$ such that for each partition $\rho$ we could choose $x_{\rho}$ and $y_{\rho}$ in some set $E_{k}$ belonging to $\rho$ such that $\left|f\left(x_{\rho}\right)-f\left(y_{\rho}\right)\right|>\varepsilon$. Define

$$
F_{\rho}=H_{x_{\rho}}-H_{y_{\rho}} \text {. }
$$

Then, $F_{\rho}$ converges boundedly to 0 , since $\left\|F_{\rho}\right\|=2$ for all $\rho$ and $F_{\rho}(E)$ $=0$ for $\rho \geqq\left\{E, E^{\prime}\right\}$. Since $\phi$ is continuous, $\lim \phi\left(F_{\rho}\right)=0$. But, since $\phi$ is linear, $\phi\left(F_{\rho}\right)=f\left(x_{\rho}\right)-f\left(y_{\rho}\right)$. Hence, $\left|\phi\left(F_{\rho}\right)\right|^{\rho}>\varepsilon$, a contradiction. So $f$ is a uniform limit of step functions. The existence of (3.4) follows from (3.3) and the continuity of $\phi$. Since step functions are bounded, $f$ is bounded.

Conversely, the integral (3.4) exists for any uniform limit of step functions $f$ and thus defines a linear functional $\phi$ on $B$. For, given any $\varepsilon>0$ there exists a partition $\rho_{\varepsilon}$ such that $\left|f\left(A_{k}\right)\right|<\varepsilon$ for all $A_{k}$ in $\rho_{\varepsilon}$. Hence, $\sum_{\rho}\left|f\left(E_{k}\right)\right|\left|F\left(E_{k}\right)\right| \leqq \sum_{\rho} \varepsilon\left|F\left(E_{k}\right)\right| \leqq \varepsilon\|F\|$ for all $\rho \geqq \rho_{\varepsilon}$. Moreover, the functional $\phi$ defined by (3.4) is continuous. For, let $F_{\alpha}$ converge boundedly to 0 . Now,

$$
\int f d F_{\alpha}=\int\left(f-f_{\rho}\right) d F_{\alpha}+\int f_{\rho} d F_{\alpha}
$$

where $f_{\rho}=\sum_{\rho} a_{k} E_{k}$ and $\left|f(x)-f_{\rho}(x)\right|<\varepsilon$ for all $x$. Hence,

$$
\left|\int f d F_{\alpha}\right| \leqq \varepsilon \| F_{a}||+\left|\sum_{\rho} a_{k} F_{a}\left(E_{k}\right)\right|
$$

and

$$
\varlimsup_{\alpha}\left|\int f d F_{\alpha}\right| \leqq \varepsilon \varlimsup_{\alpha}\left\|F_{\alpha}\right\| \leqq 2 M \varepsilon .
$$

So, $\lim _{\alpha} \phi\left(F_{a}\right)=0$.

For $f$ in $B^{*}$ define 


$$
\|f\|=\sup _{x}|f(x)|
$$

Then $B^{*}$ is a Banach space and the norm topology is equivalent to the topology of uniform convergence. With this topology a continuous linear functional on $B^{*}$ is a bounded linear functional. A bounded linear functional $\Phi$ an $B^{*}$ defines, a fortiori, a bounded additive set function on $\mathfrak{A}$. Since $\mathfrak{A}$ is fundamental in $B^{*}$, the correspondence between $\Phi$ and $F$ is biunique. Hence, $B^{* *}=B$.

For positive measures bounded convergence is equivalent to simple convergence, and hence to weak convergence over $B^{*}$. To show that bounded convergence in $B$ is, in general, stronger than weak convergence over $B^{*}$ we prove the following.

THEOREM 3. There may exist a net $\left\{F_{\alpha}\right\}$ in $B$ such that $\lim _{\alpha} \int f d F_{\alpha}$ $=0$ for every $f$ in $B^{*}$, but $F_{a}$ does not converge boundedly.

Proof. Let $X$ consist of all real-valued functions $x(t)$ on $(-\infty, \infty)$. Let $\mathfrak{U}_{\sigma}$ be the $\sigma$-algebra in $X$ generated by sets of the form

$$
I_{t}=\{x \mid x(t) \in I\}
$$

where $I$ is any interval. Let $\left\{E_{\alpha}\right\}$ be the class of all $E$ in $\mathfrak{r}_{\sigma}$ which contain the zero function 0 . The indexing set $\{\alpha\}$ is directed by defining $\alpha<\alpha^{\prime}$ whenever $E_{\alpha^{\prime}} \subset E_{\alpha}$. For each real $s$ define $x_{s}$ by

$$
x_{s}(t)= \begin{cases}0 & \text { for } t \neq s . \\ 1 & \text { for } t=s .\end{cases}
$$

Then each $E_{a}$ contains all but a countable set of $x_{s}$. Hence, we may choose $x_{s \alpha}$ in $E_{\alpha}$. Also, choose $a_{\alpha}$ such that $\lim _{\alpha} a_{\alpha}=\infty$. Finally, define

$$
F_{\alpha}=a_{\alpha}\left(H_{s_{\alpha}}-H_{0}\right)
$$

Then, $\quad\left\|F_{\alpha}\right\|=2\left|a_{\alpha}\right|$. So $\lim _{\alpha}\left\|F_{\alpha}\right\|=\infty$. For $f$ in $B^{*}, \int f d H_{0}$ exists. Therefore, $\lim _{\alpha}\left|f\left(E_{\alpha}\right)\right|=0$. Hence, $\left|f\left(x_{s}\right)-f(0)\right|>1 / n$ for only countably many values of $s$, and thus $f\left(x_{s}\right) \neq f(0)$ for only countably many values of $s$. Therefore, $f\left(x_{s \alpha}\right)=f(0)$ eventually. Since $\int f d F_{\alpha}=a_{\alpha}\left[f\left(x_{s_{\alpha}}\right)\right.$ $-f(0)], \quad \int f d F_{\alpha}=0$ eventually. A fortiori, $\lim _{\alpha} \int f d F_{\alpha}=0$.

A modification of the above example shows that the lattice operations [1] need not be continuous. Let $\mathfrak{A}$ be the algebra generated by 
sets of the form (3.10). Then, $H_{x_{n}}$ converges boundedly to $H_{0}$, where $x_{n}$ is defined by (3.11). However, $H_{x_{n}} \wedge H_{0}=0$ which does not converge to $H_{0}=H_{0} \wedge H_{0}$.

If our algebra of sets is also a $\sigma$-algebra $\mathfrak{A}_{\sigma}$, we may consider the space of countably additive set functions on $\mathfrak{A}_{\sigma}$. With the topology of bounded convergence, this space $L$ is dense in B because of (3.3). The proof of theorem 2 shows that $L^{*}=B^{*}$. However, $L$ is reflexive only with respect to the pseudo-topology on $L^{*}$ induced by the bounded convergence of sequences: $f_{n}$ converges to $f$ if $\lim _{n} f_{n}(x)=f(x)$ for each $x$ in $X$, and $\left\|f_{n}\right\|<M$ for all $n$. That $L^{* *}=L$ follows from the Lebesgue bounded convergence theorem [4].

4. The topology of regular convergence. Let $X$ be a normal Hausdorff space and $\mathfrak{A}_{\sigma}$ be the Borel sets in $X$. Let $R$ be the space of bounded, regular, signed measures on $\mathfrak{A}_{\sigma}$. That is, $F$ is in $R$ if

$$
F\left(\bigcup_{n} E_{n}\right)=\sum_{n} F\left(E_{n}\right)
$$

for every sequence $\left\{E_{n}\right\}$ of disjoint sets in $\mathfrak{H}_{\sigma}$,

$$
|F(E)|<M
$$

for all $E$ in $\mathfrak{N}_{\sigma}$, and if $\left\{A_{\alpha}\right\}$ is the class of all open sets containing $E$ and $|F|$ is the total variation of $F[1]$, then

$$
\inf _{\alpha}|F|\left(A_{\alpha}-E\right)=0 \text {. }
$$

With norm (3.2) $R$ is a Banach space and with the natural ordering a Banach lattice [1]. Since every $F$ in $R$ is the difference of two positive measures in $R$, we may define convergence for positive measures, a net of signed measures being convergent if it is the difference of two positive convergent nets.

A net $\left\{F_{a}\right)$ of positive measures in $R$ converges regularly to $F$ in $R$ if

$$
F(\text { bdy } E)=0 \text { implies } \lim _{\alpha} F_{\alpha}(E)=F(E),
$$

where bdy $E$ is the boundary of $E$. Since bdy $X$ is empty, (4.4) implies that eventually

$$
\left\|F_{a}\right\|<M \text {. }
$$

This type of convergence, under more restrictive conditions, has been considered by de La Vallée Pousisn [6].

THEOREM 4. For a net of positive measures in $R$ the following 
conditions are equivalent:

(i) $F_{a}$ converges regularly to $F$.

(ii) For every bounded, measurable function $f$ on $X$ continuous almost everywhere relative to $F, \int f d F_{\alpha}$ converges to $\int f d F$.

(iii) For every bounded, continuous function $f$ on $X, \int f d F_{a}$ converges to $\int f d F$.

(iv) $F_{\alpha}(X)$ converges to $F(X)$ and, for every closed set $E, \lim _{\alpha} F_{\alpha}(E)$ $\leqq F(E)$.

The dual space $R^{*}$ of $R$ consists of all bounded, continuous functions $f$ on $X$ with the functionals defined by (3.4). Thus, for positive measures regular convergence is equivalent to weak convergence over $R^{*}$.

Proof. Given (i) and $f$ bounded, measurable, and continuous almost everywhere relative to $F$, consider the sets $A_{t}=f^{-1}(t)$. Since $F$ is additive and bounded, and the sets $A_{t}$ are disjoint, $F\left(A_{t}\right) \neq 0$ for at most countably many values of $t$. Hence, for arbitrary $\varepsilon>0$ we can partition the range of $f$ by means of $t_{1}<t_{2}<\cdots<t_{n}$ so that $t_{k+1}-t_{k}$ $<\varepsilon$ and $F\left(A_{t_{k}}\right)=0$. Let $E_{k}=A_{t_{k}}+f^{-1}\left(t_{k}, t_{k+1}\right)$. Then bdy $E_{k} \subseteq A_{t_{k}}+A_{t_{k+1}}$ $+D$ where $D$ consists of discontinuities of $f$. Hence, $F\left(\right.$ bdy $\left.E_{k}\right)=0$. By (i), $\lim _{\alpha} F_{\alpha}\left(E_{k}\right)=F\left(E_{k}\right)$.

Let $\stackrel{\alpha}{\rho}=\left\{E_{k}\right\}$ and $f_{\rho}=\sum_{\rho} t_{k} E_{k}$. Then

$$
\begin{aligned}
& \left|\int f d F_{\alpha}-\int f d F\right| \leqq\left|\int\left(f-f_{\rho}\right) d F_{a}\right| \\
& \quad+\left|\int f_{\rho} d F_{\alpha}-\int f_{\rho} d F\right|+\left|\int\left(f_{\rho}-f\right) d F\right| \\
& \leqq \varepsilon\left\|F_{\alpha}\right\|+\left|\sum_{\rho} t_{k}\left[F_{\alpha}\left(E_{k}\right)-F\left(E_{k}\right)\right]\right|+\varepsilon\|F\| .
\end{aligned}
$$

Hence,

$$
\lim _{\alpha}\left|\int f d F_{\alpha}-\int f d F\right| \leqq 2 M \varepsilon .
$$

Since $\varepsilon$ is arbitrary,

$$
\lim _{\alpha} \int f d F_{\alpha}=\int f d F .
$$

Since a continuous function is, a fortiori, continuous almost everywhere as well as measurable, (ii) implies (iii).

Given (iii), the convergence of $F_{\alpha}(X)$ to $F(X)$ follows if we take $f(x)=1$ in (iii). Given a closed set $E$, let $A$ be any open set containing 
E. By Urysohn's lemma [7], there exists a continuous function $g$ such that $E \leqq g \leqq A$. Hence $F_{\alpha}(E) \leqq \int g d F_{\alpha}$ and $\int g d F \leqq F(A)$. Thus, (iii) gives $\lim F_{\alpha}(E) \leqq F(A)$. (iv) follows from (4.3).

Given (iv), let $E$ be any set with $F($ bdy $E)=0$. Let $\bar{E}=E \cup$ bdy $E$, a closed set. Then $F_{a}(E) \leqq F_{\alpha}(\bar{E})$, so $\lim _{\alpha} F_{\alpha}(E) \leqq \lim _{\alpha} F_{\alpha}(\bar{E}) \leqq F(E)$ by (iv). But $F(\bar{E})=F(E)$ since $F($ bdy $E)=0$. Hence

$$
\varlimsup_{\alpha} F_{\alpha}(E) \leqq F(E) .
$$

Similarly (4.9) holds for the complement $E^{\prime}$, since bdy $E^{\prime}=$ bdy $E$. Thus

$$
\lim _{\alpha} F_{\alpha}(X-E) \leqq F(X-E)
$$

Since $F_{\alpha}(X)$ converges to $F(X)$ by (iv), (4.10) gives

$$
F(X)-\lim _{\alpha} F_{\alpha}(E) \leqq F(X)-F(E) .
$$

Hence

$$
F(E) \leqq \lim _{\alpha} F_{\alpha}(E)
$$

Thus, (i) follows from (4.9) and (4.12).

Since (i) implies (iii), every bounded, continuous function $f$ on $X$ defines a continuous linear functional $\phi$ on $R$ of the form (3.4). We need only prove conversely that every $\dot{\phi}$ is of this form.

Since $H_{x}$ is regular and simple convergence implies regular convergence for positive measures, we have (3.3). So discrete measures are dense in $R$. Hence, for $\phi$ in $R^{*}$ we have (3.4) with $f$ defined by (2.4). Moreover, $f$ is continuous: If $\left\{x_{\alpha}\right\}$ is a net in $X$ converging to $x$, then $H_{x_{\alpha}}$ converges regularly to $H_{x}$. Hence, $f\left(x_{\alpha}\right)$ converges to $f(x)$. Also, $f$ is bounded: Otherwise there would exist a sequence $\left\{x_{n}\right\}$ such that $\left|f\left(x_{n}\right)\right|>2^{n}$. We could then define $F_{m}=\sum_{1}^{m}\left(1 / f\left(x_{n}\right)\right) H_{x_{n}}$. Let $F(E)$ $=\lim F_{m}(E)$. Then $F_{m}$ converges regularly to $F$ in $R$. Hence, $\phi\left(F_{m}\right)$ converges to $\phi(F)$. But, $\phi\left(F_{m}\right)=m$ which diverges, a contradiction.

5. Transition probabilities for stochastic semigroups. A system of stationary transition probabilities is a function $p(x, t, E)$ of three variables: $x$ in the sample space $X, t$ in $(0, \infty)$, and $E$ in the $\sigma$-algebra $\mathfrak{H}_{\sigma}$ in $X$ such that

(5.1) $\quad p(x, t, \quad)$ is a probability measure on $\mathfrak{A}_{\sigma}$ for each $x$ and $t$, (5.2) $p(, t, E)$ is a measurable function on $X$ for each $t$ and $E$, 
and

$$
p(y, s+t, E)=\int_{x} p(x, t, E) p(y, s, d x) .
$$

Such a system defines a family of operators $\left\{T_{t}\right\}$ on $L$ through

$$
F T_{t}(E)=\int_{X} p(x, t, E) d F(x)^{1}
$$

with the readily verified properties:

$T_{t}$ is a positive linear operator on $L$.

For $F>0, \quad\left\|F T_{t}\right\|=\|F\|$.

We call any $\left\{T_{t}\right\}$ satisfying (5.5)-(5.7) a stochastic semigroup. These have been studied by W. Feller [3].

If we try to define Markov processes abstractly by means of stochastic semigroups, we encounter the following obstacle:

THEOREM 5. There exist stochastic semigroups $\left\{T_{t}\right\}$ for which there are no transition probabilities satisfying (5.1)-(5.4).

Proof. This simple, but striking, example is due to Feller. Let $X$ be the real numbers and $\mathfrak{A}_{\sigma}$ the Borel sets of $X$. For each $F$ in $L$ let $F=F_{c}+F_{s}$ be the Lebesgue decomposition [4] where $F_{c}$ is absolutely continuous and $F_{\mathrm{s}}$ is singular. Define

$$
T_{t} E=\{x \mid x-t \in E\}
$$

for each $E$ in $\mathfrak{A}_{\sigma}$, and

$$
F T_{t}(E)=F_{c}\left(T_{-t} E\right)+F_{s}\left(T_{t} E\right) .
$$

Thus, continuous measures drift to the right and singular measures drift to the left as $t$ increases. If transition probabilities exist for this process, (5.4) implies

$$
p(x, t, E)=H_{x} T_{t}(E)=H_{x}\left(T_{t} E\right) .
$$

Hence,

$$
F T_{t}(E)=\int_{X} H_{x}\left(T_{t} E\right) d F(x)=F\left(T_{t} E\right)
$$

1 We express operators on measures as right operators and their adjoints as left operators, using the same symbol for both. 
which contradicts (5.9) if $F$ is absolutely continuous.

The preceding example suggests that we examine the adjoint operators to $\left\{T_{t}\right\}$. We see then that a system of transition probabilities defines a semigroup of operators on $L^{*}$ through

$$
T_{t} f(y)=\int_{X} f(x) p(\mathrm{y}, t, d x) .
$$

That $T_{t} f$ is a bounded, measurable function follows from the fact that $f$ is a uniform limit of step functions $f_{n}$, so $T_{t} f$ is a uniform limit of $T_{t} f_{n}$, and $T_{t} f_{n}$ is bounded and measurable. Thus, $L^{*}$ is invariant under $\left\{T_{t}\right\}$. The topological significance of this is given by the following.

THEOREm 6. A linear operator $T$ on $B$ (or $L)$ is continuous in the topology of bounded convergence if, and only if, the adjoint to $T$ defines a bounded operator on the dual space $B^{*}$ (resp. $\left.L^{*}\right)$.

Proof. Let the adjoint to $T$ be a bounded operator on $B^{*}$. Then, if $F_{\alpha}$ converges boundedly to $F$, we have $\lim _{\alpha} F_{\alpha} T E=F T E$ for all $E$, since $T E$ is in $B^{*}$. Thus, $F_{\alpha} T$ converges simply to $F T$. Since $T$ is bounded, $\left\|F_{\alpha} T\right\| \leqq\left\|F_{\alpha}\right\|\|T\|$, so $F_{\alpha} T$ converges boundedly to $F T$. Hence, $T$ is continuous with respect to bounded convergence.

Conversely, if $T$ is continuous in the topology of bounded convergence, then $T$ is bounded. To prove this we need only show that the function $u(x)=\left\|H_{x} T\right\|$ is bounded, since by (3.3), $F T=\int H_{x} T d F(x)$, so $\|F T\| \leqq \sup _{x} u(x)\|F\|$. Suppose $u(x)$ were unbounded. Then, by Zorn's lemma, there is a maximal collection $\mathfrak{M}$ of sets such that $\mathfrak{M}$ has the finite intersection property and $u(x)$ is unbounded on every finite intersection of sets in $\mathfrak{M}$. Thus, for each partition $\rho$ there exists a unique $E_{\rho}$ belonging to both $\rho$ and $\mathfrak{M}$. Choose $x_{\rho}$ and $y_{\rho}$ in $E_{\rho}$ such that $\lim u\left(x_{\rho}\right)-u\left(y_{\rho}\right)=\infty$. Let $F_{\rho}=H_{x_{\rho}}-H_{y_{\rho}}$. Then $F_{\rho}$ converges boundedly to 0 . So $F_{\rho} T$ converges boundedly to 0 . Hence, $\left\|F_{\rho} T\right\|$ is eventually bounded. But $u\left(x_{\rho}\right)-u\left(y_{\rho}\right) \leqq\left\|F_{\rho} T\right\|$, a contradiction. So $u(x)$ must be bounded.

Now, the dual of a continuous operator always exists; that is, the dual space is invariant under the adjoint operator. For, if $F_{a}$ converges to $F, F_{\alpha} T$ converges to $F T$, and so $F_{\alpha} T \phi$ converges to $F T \phi$ for each $\phi$ in the dual space. Thus, $T \phi$ is a continuous, linear functional. Hence, $T \phi$ is in the dual space.

The same proof holds for the space $L$. With an analogous argument, theorem 4 gives a similar result for the space $R$ :

THEOREM 7. A positive linear operator on $R$ is continuous in the topology of regular convergence if, and only if, the adjoint defines a 
positive operator on the dual space $R^{*}$.

We can now remedy the defect given by Theorem 5. To (5.5)(5.7) we add the condition

(5.13) $\quad T_{t}$ is continuous in the topology of bounded convergence.

THEOREM 8. To every system of transition probabilities $p(x, t, E)$ there corresponds biuniquely a stochastic semigroup $\left\{T_{t}\right\}$ satisfying (5.13) such that (5.4) holds.

Proof. We need only prove that (5.5)-(5.7) and (5.13) are sufficient, since the necessity of these conditions has already been discussed. Given $\left\{T_{t}\right\}$ we define

$$
p(x, t, E)=H_{x} T_{t} E .
$$

Condition (5.13) implies, by Theorem 6 , that $T_{t} E$ is in $L^{*}$. Hence, (5.2). (5.1) follows from (5.5), (5.6), and (5.14). (5.3) is a direct result of (5.7), (5.14), and

$$
F T_{t} E=\int_{X} H_{x} T_{t} E d F(x) .
$$

which results from $T_{t} E$ being in $L^{*}$. Finally, (5.4) follows from (5.14) and (5.15).

For the space $R$ we have the following.

THEOREM 9. Suppose (5.4) defines a stochastic semigroup of operators on $R$. Then the following conditions are equivalent:

(i ) $p(x, t, E)$ is continuous at $x$ if $p(x, t$, bdy $E)=0$.

(ii) $T_{t}$ is continuous in the topology of regular convergence.

(iii) If $f$ is a bounded, continuous function, then so is $T_{t} f$.

Proof. The equivalence of (ii) and (iii) follows directly from Theorem 7.

Given (ii), let $x_{\alpha}$ converge to $x$ in $X$. Then $H_{x_{\alpha}}$ converges regularly to $H_{x}$ so $H_{x_{\alpha}} T_{t}$ converges regularly to $H_{x} T_{t}$. Thus, $H_{x_{\alpha}} T_{t} E$ converges to $H_{x} T_{t} E$ if $H_{x} T_{t}($ bdy $E)=0$. This, through (5.14), gives (i).

Given (i), consider positive measures such that $F_{\alpha}$ converges regularly to $F$. Let $F T_{t}($ bdy $E)=0$. Then (5.4) implies $p(x, t$, bdy $E)=0$ except for $x$ in $D$, where $F(\mathrm{D})=0$. Let $f(x)=p(x, t, E)$. By (i), $f$ is continuous almost everywhere relative to $F$. Thus, condition (ii) of 
Theorem 4 applied to (5.4) gives $\lim _{\alpha} F_{\alpha} T_{t} E=F T_{t} E$. So $F_{\alpha} T$ converges regularly to $F T$, Hence, (ii).

\section{REFERENCES}

1. G. Birkhoff, Lattice theory, Amer. Math. Soc. Coll. Pub., New York, 1940.

2. N. Bourbaki, Élements de mathématiques. Intégration., Hermann, Paris, 1952.

3. W. Feller, The parabolic differential equations and the associated semi-groups of transformations, Ann. of Math., 55 (1952), 468-519.

4. P. R. Halmos, Measure theory, Van Nostrand, New York, 1950.

5. J. L. Kelley, Convergence in topology, Duke Math. J., 17 (1950), 277-283.

6. C. de la Vallée Poussin, Extension de la méthode du balayage de Poincaré et problème de Dirichlet, Note I, Ann. Inst. H. Poincaré, 2 (1932), 219.

7. W. Sierpinski, General Topology, Univ. of Toronto Press, Toronto, 1952.

RUTGERS UNIVERSITY 


\section{PACIFIC JOURNAL OF MATHEMATICS}

\section{EDITORS}

\author{
H. L. Royden \\ Stanford University \\ Stanford, California \\ E. Hewitt \\ University of Washington \\ Seattle 5 , Washington
}

R. P. Dilworth

California Institute of Technology

Pasadena 4, California

\author{
A. HorN* \\ University of California \\ Los Angeles 24, California
}

\section{ASSOCIATE EDITORS}

\author{
E. F. BECKENBACH \\ C. E. BURGESS \\ H. BUSEMANN \\ H. FEDERER
}

\author{
M. HALL \\ P. R. HALMOS \\ V. GANAPATHY IYER \\ R. D. JAMES
}

\author{
M. S. KNEBELMAN \\ I. NIVEN \\ T. G. OSTROM \\ M. M. SCHIFFER
}

J. J. STOKER

G. SZEKERES

F. WOLF

K. YOSIDA

\section{SPONSORS}

\author{
UNIVERSITY OF BRITISH COLUMBIA \\ CALIFORNIA INSTITUTE OF TECHNOLOGY \\ UNIVERSITY OF CALIFORNIA, BERKELEY \\ UNIVERSITY OF CALIFORNIA, DAVIS \\ UNIVERSITY OF CALIFORNIA, LOS ANGELES \\ UNIVERSITY OF CALIFORNIA, SANTA BARBARA \\ MONTANA STATE UNIVERSITY \\ UNIVERSITY OF NEVADA \\ OREGON STATE COLLEGE \\ UNIVERSITY OF OREGON \\ UNIVERSITY OF SOUTHERN CALIFORNIA
}

STANFORD RESEARCH INSTITUTE

STANFORD UNIVERSITY

UNIVERSITY OF UTAH

WASHINGTON STATE COLLEGE

UNIVERSITY OF WASHINGTON

AMERICAN MATHEMATICAL SOCIETY HUGHES AIRCRAFT COMPANY SHELL DEVELOPMENT COMPANY

Mathematical papers intended for publication in the Pacific Journal of Mathematics should be typewritten (double spaced), and the author should keep a complete copy. Manuscripts may be sent to any of the editors. Manuscripts intended for the outgoing editors should be sent to their successors. All other communications to the editors should be addressed to the managing editor, Alfred Horn at the University of California, Los Angeles 24, California.

50 reprints of each article are furnished free of charge; additional copies may be obtained at cost in multiples of 50 .

The Pacific Journal of Mathematics is published quarterly, in March, June, September, and December. The price per volume (4 numbers) is $\$ 12.00$; single issues, $\$ 3.50$. Back numbers are available. Special price to individual faculty members of supporting institutions and to individual members of the American Mathematical Society: $\$ 4.00$ per volume; single issues, $\$ 1.25$.

Subscriptions, orders for back numbers, and changes of address should be sent to Pacific Journal of Mathematics, c/o University of California Press, Berkeley 4, California.

Printed at Kokusai Bunken Insatsusha (International Academic Printing Co., Ltd.), No. 10, 1-chome, Fujimi-cho, Chiyoda-ku, Tokyo, Japan.

* During the absence of E. G. Straus.

PUBLISHED BY PACIFIC JOURNAL OF MATHEMATICS, A NON-PROFIT CORPORATION COPYRIGHT 1956 BY PACIFIC JOURNAL OF MATHEMATICS 


\section{Pacific Journal of Mathematics}

\section{Vol. 6, No. $3 \quad$ BadMonth, 1956}

Richard Arens and James Eells, Jr., On embedding uniform and topological spaces ............................................ 397

N. Aronszajn and Prom Panitchpakdi, Extension of uniformly continuous transformations and hyperconvex metric spaces .............. 405

Kai Lai Chung and Cyrus Derman, Non-recurrent random walks ........ 441

Harry Herbert Corson, III, On some special systems of equations . . . . . . . . . 449

Charles W. Curtis, On Lie algebras of algebraic linear transformations . . . 453

Isidore Heller, Neighbor relations on the convex of cyclic permutations . . . . 467

Solomon Leader, Convergence topologies for measures and the existence of transition probabilities..................................... 479

D. H. Lehmer, On certain character matrices ...................... 491

Michael Bahir Maschler, Minimal domains and their Bergman kernel function ........................................... 501

Wm. M. Myers, Functionals associated with a continuous transformation ................................... 517

Irving Reiner and Jonathan Dean Swift, Congruence subgroups of matrix groups ....................................... 529

Andrew Sobczyk, Simple families of lines ................... 541

Charles Standish, A class of measure preserving transformations ........ 553 Jeremiah Milton Stark, On distortion in pseudo-conformal mapping ..... 565 